\title{
On the impact of local taxes in a set cover game
}

\author{
Bruno Escoffier, Laurent Gourvès, and Jérôme Monnot \\ LAMSADE, CNRS FRE 3234, Université de Paris-Dauphine, 75775 Paris, France \\ \{escoffier, laurent.gourves, monnot\}@lamsade.dauphine.fr
}

\begin{abstract}
Given a collection $\mathcal{C}$ of weighted subsets of a ground set $\mathcal{E}$, the SET COVER problem is to find a minimum weight subset of $\mathcal{C}$ which covers all elements of $\mathcal{E}$. We study a strategic game defined upon this classical optimization problem. Every element of $\mathcal{E}$ is a player which chooses one set of $\mathcal{C}$ where it appears. Following a public tax function, every player is charged a fraction of the weight of the set that it has selected. Our motivation is to design a tax function having the following features: it can be implemented in a distributed manner, existence of an equilibrium is guaranteed and the social cost for these equilibria is minimized.
\end{abstract}

\section{Introduction}

We study a strategic game where each player should choose a facility from a set of facilities available to him. Each facility has a cost and each player must pay a tax for the facility that he has selected. This tax is a fraction of the facility's cost, and it decreases when the number of players who select the facility increases. This game is a model for many applications: facilities are services and every player is a client who selects the cheapest service.

The social cost is defined as the total cost of the facilities selected by at least one player, no matter how much the players pay. As a motivation, the cost of a facility can be an environmental cost and taxes can be a right to pollute. Then the environmental impact of the players' choice is much more important than the amount of money they pay.

In the game, no central authority can control the player's choice and minimize the social cost. Instead the players are self-interested, they all choose the facility that induces the lowest tax. We deal with the case where taxes are locally defined on the facilities (independently on the players' choices for other facilities) so it can be implemented in a completely distributed manner. Local tax functions induce a game among the players. The question posed in this article is "Which local tax function minimizes the social cost?". Assuming that the game's outcome is an equilibrium, we study the price of anarchy (PoA in short) which is the worst case ratio between the social cost of a equilibrium, and the optimal social cost [1]. In particular we consider pure strategy Nash equilibria and its robust refinements to strong equilibria and $k$-strong equilibria.

\footnotetext{
* This work is supported by French National Agency (ANR), project COCA ANR-09-
} JCJC-0066-01. 
We first study a tax function where the cost of a facility is evenly shared by the players who select it. It is fair (the users of the a facility are treated equally) and budget balanced (the taxes cover the total cost). Next we investigate local tax functions which are fair, non-negative and monotone non-increasing in the number of players who select it. Any local tax function of this kind encourages the sharing of the facilities and it guarantees the existence of a pure strategy strong equilibrium.

\section{Definitions, related work and summary of results}

Set Cover. The situation described in introduction is usually modeled as a SET COVER problem. Given a set $\mathcal{E}=\left\{e_{1}, \ldots, e_{n}\right\}$ of $n$ elements, a collection $\mathcal{S}=$ $\left\{S_{1}, \ldots, S_{m}\right\}$ of $m$ subsets of $\mathcal{E}$ such that $\bigcup_{j=1}^{m} S_{j}=\mathcal{E}$ and a weight function $w: \mathcal{S} \rightarrow \mathbb{R}_{+}$, the problem is to find $X \subseteq \mathcal{S}$ such that every element in $\mathcal{E}$ belongs to at least one member of $X$ and $\sum_{S_{j} \in X} w\left(S_{j}\right)$ is minimum. In the following we sometimes write $w_{j}$ (resp. $s_{j}$ ) to denote $w\left(S_{j}\right)$ (resp. $\left|S_{j}\right|$ ).

We study a SET COVER game defined upon the SET COVER problem. A facility $j$ is associated with each set $S_{j} \in \mathcal{S}$. Each element $e_{i} \in \mathcal{E}$ is controlled by a player $i$ who wants $e_{i}$ to be covered by a set of $\mathcal{S}$. The set of facilities $\{1, \ldots, m\}$ and the set of players $\{1, \ldots, n\}$ are respectively denoted by $M$ and $N$. Each player $i \in N$ has a strategy set $\Sigma_{i}$ defined as $\left\{j \in M: e_{i} \in S_{j}\right\}$. We denote by $\Sigma=\Sigma_{1} \times \cdots \times \Sigma_{n}$ the set of all states (or strategy profiles). The $i$-th coordinate of $a \in \Sigma$, denoted by $a_{i}$, is the action of $i$ (actions are singletons). The congestion (or load) of a facility $j$ is the number of players who want their element to be covered by $S_{j}$. It is denoted by $\ell_{j}(a)$ and defined as $\left|\left\{i \in N: a_{i}=j\right\}\right|$.

In the general tailored model, there is one function $C$ which depends on locally available values: $s_{j}, w_{j}$ and $\ell_{j}(a)$ for a given state $a$. This function $C$ is unique, public and used locally by every facility. $C\left(s_{j}, w_{j}, \ell_{j}(a)\right)$ is the tax that every player who has selected $j$ must pay. We assume that $C$ exhibits economies of scale, i.e. $C$ is a monotone non increasing function of $\ell_{j}(a)$. In addition, $C$ is non negative (players are not paid to select a facility). The goal in this model is to find a function that minimizes the social cost. If the taxes paid by the agents do not cover the cost of all selected facilities then we interpret it as the introduction of subsidies.

In the Fair balanced model, the cost of a facility is evenly shared by the players that chose this facility: $C\left(s_{j}, w_{j}, \ell_{j}\right)=w_{j} / \ell_{j}$. This function is a natural particular case of the tailored model. It corresponds to situations where we want a fair and budget balanced cost (the value of a state equals the sum payments of the agents, while this property does not necessary holds for other tax functions in the tailored model).

Finally, the tax that player $i$ must pay under the state $a$ is $C\left(s_{a_{i}}, w_{a_{i}}, \ell_{a_{i}}(a)\right)$ but we often write $c_{i}(a)$ instead.

Solutions, existence and ratios. Much of the research in computational game theory has focused on the Nash equilibrium (NE in short). It is a state in which no single player can deviate and benefit. A strong equilibrium (SE in short) is a state in which no coalition of players can deviate and benefit [2]. This refinement 
of the NE is then more robust. Given $a \in \Sigma, i^{\prime} \in N$ and $j^{\prime} \in \Sigma_{i^{\prime}},\left(\left.a\right|_{i^{\prime}} j^{\prime}\right)$ denotes the strategy profile where every player $i \in N \backslash\left\{i^{\prime}\right\}$ plays $a_{i}$ whereas $i^{\prime}$ plays $j^{\prime}$. A pure strategy Nash equilibrium is a state $a \in \Sigma$ where $\forall i \in N, \forall j \in \Sigma_{i}$, $c_{i}(a) \leq c_{i}\left(\left.a\right|_{i} j\right)$. Pure means that every player chooses a strategy deterministically and since we only consider pure strategies, we often omit the adjective 'pure'.

Given $N^{\prime} \subseteq N$ and $a, b \in \Sigma,\left(\left.a\right|_{N^{\prime}} b\right)$ is the strategy profile where $i$ plays $a_{i}$ (resp. $i$ plays $b_{i}$ ) if $i \in N \backslash N^{\prime}$ (resp. if $i^{\prime} \in N^{\prime}$ ). A strong equilibrium is a state $a \in \Sigma$ where there is no coalition $N^{\prime} \subseteq N$ and $b \in \Sigma$ such that $\forall i \in N^{\prime}, c_{i}\left(\left.a\right|_{N^{\prime}} b\right)<c_{i}(a)$. A $k$-strong equilibrium is defined similarly for coalitions involving at most $k$ players.

The set of Nash equilibria (resp. strong equilibria, $k$-strong equilibria) of a game $\Gamma$ (the SET COVER game in our case) is denoted by $N E(\Gamma)$ (resp. $S E(\Gamma), k S E(\Gamma)$ ). In particular $S E(\Gamma) \subseteq k S E(\Gamma) \subseteq N E(\Gamma) \subseteq \Sigma$.

A state is said optimal if it minimizes the social cost. The minimal social cost is denoted by $O P T$. We denote by $N A S H$ (resp. STRONG, $k S T R O N G$ ) the social cost of a given a Nash equilibrium (resp. strong equilibrium, $k$-strong equilibrium).

The price of anarchy (PoA) is a widely accepted performance measure of decentralized systems which relies on Nash equilibria [1]. It was generalized to $k$-strong equilibria and named the $k$-strong price of anarchy ( $k-\mathrm{SPoA}$ in short) [3]. The $k$-SPoA is the worst case ratio between the weight of a pure $k$-strong equilibrium (assuming one exists) and the optimum, over all instances of a game. The PoA and the strong price of anarchy (SPoA in short) correspond to the cases $k=1$ and $k=n$ respectively.

Related work. The SET COVER game is a congestion game where strategies are singletons. Rosenthal [4] showed that every congestion game possesses a pure strategy NE. The existence of SE in congestion games was first studied by Holzman and Law-Yone [5] who focused on situations where congestion has a negative effect on the players' utility (monotone-decreasing congestion games). After that, Rozenfeld and Tennenholtz [6] completed the study for monotone-increasing congestion games where congestion has a positive effect on the players' utility. In particular they prove that monotone-increasing congestion games where strategies are singletons always has a pure strategy strong equilibrium. In addition, this SE can be computed efficiently. The SET COVER game under the tailored model is a monotone-increasing congestion game so it always possesses a SE.

In [7], Anshelevich et al. study a connection game consisting of a network (an edge-weighted graph) and a pair $\left(s_{i}, t_{i}\right)$ of nodes for each player $i$. A player's strategy is a set of edges that form an $s_{i}-t_{i}$ path. In the unweighted case (all players have the same importance), the cost of each selected edge is shared equally by its users, i.e. if $k$ agents use an edge of cost $w$ in their strategy then each of these agents pays a share of $w / k$. The SET COVER game under the fair balanced model is a particular case of Anshelevich et al.'s connection game if the network is as follows: we are given a supersource $x$, a node $y_{j}$ and an edge $\left(x, y_{j}\right)$ of cost $w_{j}$ for each set $S_{j}$, a node $z_{i}$ for each element $e_{i}$ and an edge $\left(y_{j}, z_{i}\right)$ of cost 0 iff $e_{i} \in S_{j}$. Each player $i$ wants to connect $x$ to $z_{i}$. The PoA of Anshelevich et al.'s connection game is $n$ (the number of players) and the price of stability (the ratio of the best 
Nash equilibrium relative to the social optimum) is $H(n)=O(\log n)[7]$ where $H(n)=1+1 / 2+\cdots+1 / n$.

The connection game is also studied by Epstein et al. [8] who give topologic conditions for the existence of a strong equilibrium and bounds on the $k$-SPoA. In particular, when all players have the same source but not necessarily the same sink (single source), there is a strong equilibrium if the network is a series parallel graph [8]. This result applies for the "network" representation of the SET COVER game given above. For the $k$-strong price of anarchy of the connection game Epstein et al. prove that $\max \left\{\frac{n}{k}, H(n)\right\} \leq k$-SPoA $\leq \frac{n}{k} H(k)$. Therefore the PoA is $n$ and the SPoA is $H(n)$.

In this paper we consider the fair balanced model like in $[7,8]$ but we also investigate general local taxes (tailored model). The goal is to find one that induces the best system's efficiency. In other words, we aim at mitigating the system's deterioration due to selfish and uncoordinated behavior. In this line, Christodoulou et al. introduced the notion of coordination mechanism [9]. Recently coordination mechanisms received a lot of attention, in particular for scheduling games.

Finally the SET COVER game under consideration in this paper is close to the model studied by Buchbinder et al. [10]. A central authority encourages the purchase of resources by offering subsidies that reduce their price: every player who has selected resource $j$ pays $\left(w_{j}-\sigma_{j}\right) / \ell_{j}$ where $\sigma_{j}$ is the subsidy associated with $j$. Hence the fair balanced model corresponds to the case where $\sigma_{j}=0$ for all $j$. The main differences with our model are that the players are introduced sequentially by an adversarial scheduler and the total amount of subsidies offered is bounded by the amount of non refundable taxes collected when a player purchases a new set.

Summary of results. We study in Section 3 the $k$-SPoA of the SET COVER game in the fair balanced model. We prove that $k$-SPoA $=H(k)-1+\frac{\Delta}{k}$ where $\Delta$ is the maximum size of a set. We deduce that $\mathrm{PoA}=\Delta$ and $\mathrm{SPoA}=H(\Delta)$. We also deduce that $k$-SPoA $=H(k)-1+\frac{n}{k}, \mathrm{PoA}=n$ and $\mathrm{SPoA}=H(n)$ where $n$ is the number of players. These bounds are tight. When $k=1$ and $k=n$, they meet the previous results given in $[7,8]$. However, when $1<k<n$, we get a more accurate value of the $k$-SPoA, i.e. $H(k)-1+\frac{n}{k}$ instead of the $\frac{n}{k} H(k)$ given by Epstein et al. [8].

In Section 4, we study the tailored model. We first show that the PoA can reduce by more than $1 / 3$ using a function that encourages players to choose big sets. On the other hand, we also show in this section strong lower bounds valid for any tax function. Bounds on the PoA obtained in the tailored model are of the same order as those obtained with the fair balanced model (see Table 1 for a summary of the results given in this article). Then local taxes cannot (in the model dealt with here) considerably reduce the social cost (compared to a fair and balanced division of the cost).

Finally the case of uniform weights is studied. We mainly prove a lower bound of $n / 4$ and an almost matching upper bound of $n / 4+1 / 2+1 / 4 n$ for the PoA. These bounds hold in the tailored model whereas $\mathrm{PoA}=n$ in the fair balanced model.

Due to space limitations, some proofs are omitted. 


\begin{tabular}{|c||c|c|c||c|c|c|}
\hline \multicolumn{1}{|c||}{} & \multicolumn{3}{c||}{$n$} & \multicolumn{3}{|c|}{$\Delta$} \\
\hline & PoA & SPoA & $k$-SPoA & PoA & SPoA & $k$-SPoA \\
\hline UB & $0.66 n$ & $H(n)$ & $H(k)-1+\frac{n}{k}$ & $\Delta$ & $H(\Delta)$ & $H(k)-1+\frac{\Delta}{k}$ \\
LB & $0.5 n$ & $\frac{\ln (n)}{2}+O(1)$ & $\max \left(\frac{\ln (n)}{2}+O(1), \frac{n}{2 k}\right)$ & $\Delta$ & $H(\Delta)$ & $H(k)-1+\frac{\Delta}{k}$ \\
\hline
\end{tabular}

${ }^{a}$ when $k \leq \Delta$. When $k>\Delta$, the $k$-SPoA is $H(\Delta)$.

Table 1. Upper and lower bounds on the $k$-SPoA as a function of (left part) $n$ and (right part) $\Delta$ in the tailored model.

\section{Fair balanced model}

We give in Theorem 1 the $k$-SPoA of the SET COVER game. Note that w.l.o.g., we can assume that $k \leq \Delta$ since any coalition $N^{\prime}$ can be decomposed into coalitions $N_{1}^{\prime}, \ldots N_{p}^{\prime}$ where each player of $N_{i}^{\prime}$ will play the same set $S_{j_{i}}$ for $i=1, \ldots, p$. The coalition $N^{\prime}$ strictly decreases the cost of each player in $N^{\prime}$ iff for every $i \in$ $\{1, \ldots, p\}$, the coalition $N_{i}^{\prime}$ strictly decrease the cost of each player in $N_{i}^{\prime}$. Now, since $\left|N_{i}^{\prime}\right| \leq\left|S_{j_{i}}\right| \leq \Delta$, the result follows.

Theorem 1. For any $k \in\{1, \ldots, \Delta\}$, the $k-S P O A$ of the SET COVER game is $H(k)-1+\frac{\Delta}{k}$.

Proof. Suppose that the players have reached a $k$-strong equilibrium $a$, i.e., there is no coalition $N^{\prime} \subseteq N\left(\left|N^{\prime}\right| \leq k\right)$ and $b \in \Sigma$ such that $c_{i}\left(\left.a\right|_{N^{\prime}} b\right)<c_{i}(a)$ for all $i \in N^{\prime}$.

Given $j \in[1 . . m]$ and $x \in\left[1 . .\left|S_{j}\right|\right], h(x, j)$ designates the player in $S_{j}$ with the $x$-th largest cost. Hence $c_{h(1, j)} \geq c_{h(2, j)} \geq \ldots \geq c_{h\left(\left|S_{j}\right|, j\right)}$ holds. Ties are broken arbitrarily. Let us show that

$$
c_{h(x, j)} \leq \frac{w_{j}}{\min \{x, k\}}
$$

holds for all $j \in[1 . . m]$ and $x \in\left[1 . .\left|S_{j}\right|\right]$. By contradiction, assume that there are $j_{0} \in[1 . . m]$ and $x_{0} \in\left[1 . .\left|S_{0}\right|\right]$ such that $c_{h\left(x_{0}, j_{0}\right)}>\frac{w\left(S_{j_{0}}\right)}{\min \left\{x_{0}, k\right\}}$. By assumption we get:

$$
\forall x \in\left\{1, \ldots, \min \left\{x_{0}, k\right\}\right\}, \quad c_{h\left(x, j_{0}\right)}>\frac{w\left(S_{j_{0}}\right)}{\min \left\{x_{0}, k\right\}}
$$

since $c_{h\left(x, j_{0}\right)} \geq c_{h\left(\min \left\{x_{0}, k\right\}, j_{0}\right)} \geq c_{h\left(x_{0}, j_{0}\right)}$. Now, let us consider the coalition $N^{\prime}=$ $\left\{h\left(x, j_{0}\right): x=1, \ldots, \min \left\{x_{0}, k\right\}\right\} \cap\left\{i \in N: a_{i} \neq j_{0}\right\}$ (where $a$ is the $k$-strong equilibrium). Obviously, $\left|N^{\prime}\right| \leq k$ and we get $N^{\prime} \neq \emptyset$ since otherwise $\left\{h\left(x, j_{0}\right)\right.$ : $\left.x=1, \ldots, \min \left\{x_{0}, k\right\}\right\} \subseteq S_{j_{0}}$ and then, the cost of each player in $\left\{h\left(x, j_{0}\right): x=\right.$ $\left.1, \ldots, \min \left\{x_{0}, k\right\}\right\}$ will be at most $\frac{w\left(S_{j_{0}}\right)}{\min \left\{x_{0}, k\right\}}$, contradiction with inequality (2). If all the players in $N^{\prime}$ change their mind, form a coalition and simultaneously decide to be covered by $S_{j_{0}}$ then their individual cost would be at most $\frac{w\left(S_{j_{0}}\right)}{\min \left\{x_{0}, k\right\}}$. We deduce that the solution is not a $k$-strong equilibrium, contradiction. 
Using (1) and the harmonic function $H(p):=\sum_{i=1}^{p} 1 / i$, we deduce that

$$
\begin{aligned}
\sum_{i \in S_{j}} c_{i} & \leq\left(\sum_{i=1}^{\min \left\{\left|S_{j}\right|, k\right\}} \frac{1}{i}+\frac{\left|S_{j}\right|-\min \left\{\left|S_{j}\right|, k\right\}}{\min \left\{\left|S_{j}\right|, k\right\}}\right) w_{j} \\
& =\left(H\left(\min \left\{\left|S_{j}\right|, k\right\}\right)-1+\frac{\left|S_{j}\right|}{\min \left\{\left|S_{j}\right|, k\right\}}\right) w_{j} \\
& \leq\left(H(\min \{\Delta, k\})-1+\frac{\Delta}{\min \{\Delta, k\}}\right) w_{j}
\end{aligned}
$$

holds for all $j \in\{1, \ldots, m\}$ since $\left|S_{j}\right| \leq \Delta$. Let $X \subseteq \mathcal{S}$ be an optimal solution to the underlying SET COVER problem with value $O P T=\sum_{S_{j} \in X} w_{j}$. Summing up inequalities (3) for the sets in $X$, we obtain:

$$
\sum_{S_{j} \in X} \sum_{i \in S_{j}} c_{i} \leq \sum_{S_{j} \in X}\left(H(\min \{\Delta, k\})-1+\frac{\Delta}{\min \{\Delta, k\}}\right) w_{j}
$$

On the one hand $k S T R O N G=\sum_{i \in N} c_{i} \leq \sum_{S_{j} \in X} \sum_{i \in S_{j}} c_{i}$ holds because $X$ is feasible, i.e. each element $e \in \mathcal{E}$ is covered by at least one set in $X$. On the other hand, we get: $\sum_{S_{j} \in X}\left(H(\min \{\Delta, k\})-1+\frac{\Delta}{\min \{\Delta, k\}}\right) w_{j}=(H(\min \{\Delta, k\})-1+$ $\left.\frac{\Delta}{\min \{\Delta, k\}}\right) O P T$. A tight example (omitted for space reason) exists.

From Theorem 1, we deduce that the PoA of the SET COVER game is $\Delta$ (set $k=1)$ and the SPoA of the SET COVER game is $H(\Delta)($ set $k=\Delta)$.

As a corollary, since $\Delta \leq n$, we get that the $k$-SPoA is at most $H(k)-1+\frac{n}{k}$, and hence that the PoA is at most $n$ and the SPoA at most $H(n)$. These bounds are actually tight because $\Delta=n$ in the example showing the lower bound in the proof of Theorem 1 (omitted for space reason).

Corollary 1. For any $k \in\{1, \ldots, n\}$, the $k-S P o A$ of the SET COVER game is $H(k)-1+\frac{n}{k}$.

\section{Tailored model: finding the right taxes}

The goal is to find a function $C$ which minimizes the social cost. $C$ is a non negative function monotone non increasing with $\ell_{j}(a)$. Equilibria are defined with respect to the taxes paid by players but the social cost remains the total weight of the selected sets. In contrast with the fair balanced model, we do not impose that the taxes cover the whole cost.

\subsection{Improvement on the PoA}

We use a function where the players are encouraged to select large sets (function decreasing in $s_{j}=\left|S_{j}\right|$ ) with a lot of other players (function decreasing in $\ell_{j}(a)$ ). We first show a lemma satisfied by these functions, and then study a particular class of functions that leads to interesting bounds (Lemma 2 and Theorem 2). 
Fix a cost function and suppose that we have a NE strategy profile $a$ (for this cost function). After a possible relabeling, we denote by $Y=\left\{S_{1}, S_{2}, \cdots, S_{t}\right\}$ the set of sets chosen by at least one player with $s_{1} \geq s_{2} \geq \cdots \geq s_{t}$. For the sake of clarity, suppose that $a_{i}=i$ for $i \leq t$ (i.e. player $i$ chooses $S_{i}$ ).

Lemma 1. Let $c$ be a cost function which is decreasing in $\ell_{j}(a)$. Then, for any $i \in\{1,2, \cdots, t\}, s_{i} \leq n+1-i$.

Proof. Suppose that there exists $i$ such that $s_{i} \geq n+2-i$. Since $s_{k}$ are sorted in non increasing order, for any $k \leq i s_{k} \geq n+2-i$. Then fix some $j \leq i$. Let $N^{\prime}=\{1, \cdots, i\} \backslash\{j\}$. Since $s_{j} \geq n+2-i, s_{j}+\left|N^{\prime}\right| \geq n+1$ and consequently there exists a player $k \in N^{\prime}$ with $e_{k} \in S_{j}$. Then, since the profile is a NE, $c_{k}(a) \leq c_{k}\left(\left.a\right|_{k} j\right)$. Since $c$ is decreasing in $\ell_{j}(a), c_{k}(a)<c_{j}(a)$.

In the strategy profile $a$, for any $j \leq i$ there exists a $k \leq i$ such that $c_{k}(a)<$ $c_{j}(a)$. This is obviously impossible.

We now study a particular class of local tax functions $\frac{w_{a_{i}}}{g\left(s_{a_{i}}\right)+\epsilon g\left(\ell_{a_{i}}(a)\right)}$ where $g$ is a positive and increasing function and $\epsilon>0$. Fix an optimal Set Cover $\left\{S_{1}^{*}, S_{2}^{*}, \cdots, S_{t^{*}}^{*}\right\}$, and let $s_{j}^{*}=\left|S_{j}^{*}\right|$.

Lemma 2. Let the tax function be $\frac{w_{a_{i}}}{g\left(s_{a_{i}}\right)+\epsilon g\left(\ell_{a_{i}}(a)\right)}$ where $g$ is a positive and increasing function. Then:

$$
N A S H \leq(1+\epsilon) \sum_{j=1}^{t^{*}}\left(w\left(S_{j}^{*}\right) \frac{\sum_{i=1}^{s_{j}^{*}} g(n+1-i)}{g\left(s_{j}^{*}\right)}\right)
$$

Proof. As previously, suppose that player $i$ chooses $S_{i}(i \leq t)$ and let $\lambda(j)$ (for $\left.j \leq t^{*}\right)$ be the set of players among $\{1,2, \cdots, t\}$ which belong to $S_{j}^{*}(\lambda(j)$ is possibly empty).

Since $g$ is increasing, the $\operatorname{cost} c_{i}(a)$ associated to player $i$ verifies:

$$
c_{i}(a)=\frac{w\left(S_{i}\right)}{g\left(s_{i}\right)+\epsilon g\left(\ell_{i}(a)\right)} \geq \frac{w\left(S_{i}\right)}{(1+\epsilon) g\left(s_{i}\right)}
$$

On the other hand, since the profile $a$ is a Nash equilibrium (and since $g$ is non negative), if $e_{i}$ belongs to the set $S_{j}^{*}$ :

$$
c_{i}(a) \leq c_{i}\left(\left.a\right|_{i j} j\right) \leq \frac{w\left(S_{j}^{*}\right)}{g\left(s_{j}^{*}\right)}
$$

From Equations (4) and (5) we get:

$$
w\left(S_{i}\right) \leq(1+\epsilon) w\left(S_{j}^{*}\right) \frac{g\left(s_{i}\right)}{g\left(s_{j}^{*}\right)}
$$

where $j$ is such that $e_{i} \in S_{j}^{*}$. Summing up this inequality for all players $i \in \lambda(j)$, we get: 


$$
\forall j \in\left\{1,2, \cdots, t^{*}\right\}, \sum_{i \in \lambda(j)} w\left(S_{i}\right) \leq(1+\epsilon) w\left(S_{j}^{*}\right) \frac{\sum_{i \in \lambda(j)} g\left(s_{i}\right)}{g\left(s_{j}^{*}\right)}
$$

Since $s_{i} \leq n+1-i$ (thanks to Lemma 1$)$, and since $g\left(s_{i}\right)$ is non negative and increasing, we get:

$$
\sum_{i \in \lambda(j)} g\left(s_{i}\right) \leq \sum_{i=1}^{|\lambda(j)|} g(n+1-i) \leq \sum_{i=1}^{s_{j}^{*}} g(n+1-i)
$$

Since the sets $S_{j}^{*}$ cover all the elements, each player $i$ belongs to at least one $\lambda(j)$. Then, summing Inequality (6) for $j \in\left\{1,2, \cdots, t^{*}\right\}$, we get using (7):

$$
\sum_{i=1}^{t} w\left(S_{i}\right) \leq \sum_{j=1}^{t^{*}} \sum_{i \in \lambda(j)} w\left(S_{i}\right) \leq(1+\epsilon) \sum_{j=1}^{t^{*}}\left(w\left(S_{j}^{*}\right) \frac{\sum_{i=1}^{s_{j}^{*}} g(n+1-i)}{g\left(s_{j}^{*}\right)}\right)
$$

We are now able to find the PoA associated to the cost function under consideration.

Theorem 2. Let the tax function be $c_{i}(a)=\frac{w_{a_{i}}}{g\left(s_{a_{i}}\right)+\epsilon g\left(\ell_{a_{i}}(a)\right)}$ where $g$ is a positive and increasing function. Then

$$
\frac{f(n)}{1+\epsilon} \leq P o A \leq(1+\epsilon) f(n)
$$

where $f(n)=\max _{i=1,2 \cdots, n}\left\{\frac{\sum_{k=1}^{i} g(n+1-k)}{g(i)}\right\}$.

Proof. The upper bound is a straightforward consequence of Lemma 2.

For the lower bound, consider the following instance with $n$ players and $p+2$ resources where $S_{i}=\{i, i+1, \cdots, n\}$ for $i=\{1,2, \cdots, p+1\}$ and $S_{0}=\{1,2, \cdots, p\}$. We set $w_{p+1}=0$ and fix the weights in such a way that the strategy where player $i \leq p$ chooses $S_{i}$ (and player $i>p$ chooses $S_{p+1}$ ) is a NE. We fix for $i=1,2, \cdots, p$ :

$$
w\left(S_{i}\right)=g\left(s_{i}\right)+\epsilon g(1)=g(n+1-i)+\epsilon g(1)
$$

which ensures that $\frac{w\left(S_{i}\right)}{g\left(s_{i}\right)+\epsilon g(2)} \geq \frac{w\left(S_{i+1}\right)}{g\left(s_{i+1}\right)+\epsilon g(1)}$ for $i=1,2, \cdots, p-1$. In particular, the cost associated to player $i$ is 1 if $i \leq p$ and 0 if $i \geq p+1$. We set $w\left(S_{0}\right)=g(p)+\epsilon g(1)$, ensuring that players have no incentive to deviate.

Then we have a NE of value $\sum_{i=1}^{p}(g(n+1-i)+\epsilon g(1))$ while the solution consisting of taking only $S_{0}$ and $S_{p+1}$ is a Set Cover of value $g(p)+\epsilon g(1)$. Then the PoA is such that:

$$
P o A \geq \frac{p \epsilon g(1)+\sum_{i=1}^{p} g(n+1-i)}{\epsilon g(1)+g(p)} \geq \frac{\sum_{i=1}^{p} g(n+1-i)}{g(p)(1+\epsilon)}
$$

where the last inequality holds since $g$ is increasing. 
Note that the lower bound would hold for any cost function $\frac{w_{j}}{g\left(s_{j}\right)+h\left(\ell_{j}\right)}$ where $g$ and $h$ are increasing functions.

Now we derive from Theorem 2 a PoA smaller than $n$ by choosing interesting functions $g$. Since $g$ is non decreasing, we have:

$$
\int_{k=n-i}^{n} g(x) d x \leq \sum_{k=1}^{i} g(n+1-k) \leq \int_{k=n+1-i}^{n+1} g(x) d x
$$

If we choose $g(i)=i^{\alpha}$ for some $\alpha>0$, we get:

$$
\frac{n^{\alpha+1}-(n-i)^{\alpha+1}}{\alpha+1} \leq \sum_{k=1}^{i} g(n+1-k) \leq \frac{(n+1)^{\alpha+1}-(n+1-i)^{\alpha+1}}{\alpha+1}
$$

Studying this expression, if we choose $\alpha=1 / 2$, then we get a PoA equivalent to $\beta n$ (up to a factor $1+\epsilon$ ) where $\beta=\frac{3-\sqrt{3}}{\sqrt{2} 3^{1 / 4}}<0.69$. The best choice for $\alpha$ is actually $\alpha \simeq 0.643$ which gives a PoA equivalent to $\gamma n$ (up to a factor $1+\epsilon$ ) where $\gamma \simeq 0.660$.

\subsection{Lower bounds}

We first deal with lower bounds which depend on $\Delta$ (and $k$ for $k$-SPoA). As shown in Section 3, with the fair division of the cost we get a PoA of $H(k)-1+\frac{\Delta}{k}(k \leq \Delta)$. Here, we show that despite the quite important relaxation we consider (choosing the tax function), the social cost cannot be lowered down since no cost function can lead to a better $k$-SPoA.

Before giving lower bounds, we state a preliminary lemma (proof omitted).

Lemma 3. Suppose that there exist $d \geq 1, k \geq s \geq 1$ and $w>0$ such that $C(d, 0, d)>C(s, w, s)$. Then the $k-S P o A$ of the SET COVER game is unbounded.

It follows that every non-increasing tax function inducing a bounded $k$-SPoA satisfies $C(d, 0, d) \leq C(s, w, \ell)$ where $1 \leq \ell \leq s$ and $w>0$. Now we are ready to prove lower bounds.

Proposition 1. Under the tailored model, and for any $k \in\{1, \cdots, \Delta\}$, the $k-S P o A$ of the SET COVER game is at least $H(k)-1+\frac{\Delta}{k}$.

Proof. Let $k \in\{1, \cdots, \Delta\}$. We consider an array of $(\Delta+1) \times(\Delta !)$ elements that we arrange in $\Delta+1$ lines and $\Delta$ ! columns. For $q=1, \cdots, \Delta$ !, a set $S_{q}$ contains the elements of column $q$ from line 1 to line $\Delta$. These sets are called vertical. For $l=1, \cdots, k-1$, line $l$ is partitioned into $(\Delta !) / l$ sets of size $l$. For $l=k, \cdots, \Delta$, line $l$ is partitioned into $(\Delta !) / k$ sets of size $k$. Line $\Delta+1$ is partitioned into $(\Delta !) / \Delta$ sets of size $\Delta$. These sets are called horizontal. Actually, we complete each such horizontal set by adding (at random) elements from line $\Delta+1$ in such a way that each horizontal set has size $\Delta$. Hence, for sets from line $l<k$ we add $\Delta-l$ elements, 
and for sets from line $l, k \leq l \leq \Delta$, we add $\Delta-k$ elements. Vertical sets have weight one, and horizontal sets of line between 1 and $\Delta$ have weight one, horizontal sets of line $\Delta+1$ have weight 0 .

Let us consider the state where every element of line $\Delta+1$ decides to be covered by the horizontal set where it appears (of weight 0 ), while in the other lines every element decides to be covered by the (unique) horizontal set where it appears. Hence the cost incurred by an element of line $l$ is $C(\Delta, 1, l)$ for $l<k, C(\Delta, 1, k)$ for $k \leq l \leq \Delta$, and $C(\Delta, 0, \Delta)$ for $l=\Delta+1$. As a consequence of Lemma 3, we can assume that the elements of a set of weight 0 choose this set. If $r \leq k$ elements move to the same vertical set then at least one of them does not benefit since the new cost is $C(\Delta, 1, r)$ while the lowest previous cost, thanks to the fact that $C$ is non increasing with $\ell_{j}(a)$, was at most $C(\Delta, 1, r)$. Then the state is a $k$-strong equilibrium. The optimum solution (made of all vertical sets plus the sets of weight 0 ) has weight $\Delta$ !, while the $k$-strong equilibrium we found has weight $\left(\sum_{l=1}^{k-1} \frac{\Delta !}{l}\right)+\frac{\Delta !}{k}(\Delta+1-k)=\Delta !\left(H(k)-1+\frac{\Delta}{k}\right)$.

For $k=\Delta$ (and more generally for $k \geq \Delta$ ) this gives $H(\Delta)$. For $k=1$, it shows that the PoA is at least $\Delta$.

Let us consider now lower bounds in terms of $n$ (and $k$ for $k$-SPoA). The instance given in Proposition 1 does not give a good lower bound since $n$ is very big (the lower bound is $H(k)$ for $k \leq \Delta$ but $H(\Delta)$ for $k \geq \Delta$, which is not good since $\Delta$ is very small with respect to $n$ ). In the following proposition, we show an almost tight lower bound of $\max \left(\frac{\ln (n)}{2}+O(1),\left\lfloor\frac{n+k}{2 k}\right\rfloor\right)$. Remember that the upper bound is $H(k)-1+\frac{n}{k}$, which is of order of $\ln (n)$ if $k \geq n / \ln (n)$ (as the lower bound) and of order of $n / k$ if $k \leq n / \ln (n)$ (as the lower bound) ${ }^{1}$.

For $k=1$, the lower bound is $\left\lfloor\frac{n+1}{2}\right\rfloor \geq \frac{n}{2}$, which is quite close to the PoA of $0.66 n$ obtained in Section 4.1.

Proposition 2. Under the tailored model, and for any $k \in\{1, \cdots, \Delta\}$, the $k-S P o A$ of the SET COVER game is at least $\max \left\{\frac{\ln (n)}{2}+O(1),\left\lfloor\frac{n+k}{2 k}\right\rfloor\right\}$.

Proof. Fix some $n$. To get the first lower bound, we adapt the instance given in Proposition 1 by trying to reduce the number of elements. Let $t$ be the largest integer such that $t^{2} \leq n$. We consider a square of $t$ times $t$ elements, and $n-t^{2}$ extra elements. We consider $t$ vertical sets (the columns of the square) of weight 1 , and in line $i\left\lfloor\frac{t}{i}\right\rfloor$ disjoint sets of size $i$. We add to these sets $t-i$ elements (at random) from line $t$. We group all the elements not covered by these horizontal sets (including the $n-t^{2}$ extra ones) in one set $S$ of weight 0 . All other sets have weight 1 . Note that all the sets (except possibly $S$ ) have size $t$.

We consider the solution where each player in set $S$ chooses $S$, players in line $t$ choose the set which contains this line, and players in line from 1 to $t-1$ choose the unique horizontal set to which they belong. By a similar reasoning, this is a strong equilibrium, of value $\sum_{i=1}^{t}\left\lfloor\frac{t}{i}\right\rfloor$. However $\sum_{i=1}^{t}\left\lfloor\frac{t}{i}\right\rfloor \geq \sum_{i=1}^{t}\left(\frac{t}{i}-1\right) \geq t(H(t)-1)$.

\footnotetext{
${ }^{1}$ The largest ratio between these bounds is 4 , when $k$ is of order of $n / \ln (n)$.
} 
The solution consisting of set $S$ plus the vertical sets has weight $t$, hence the ratio is at least $H(t)-1=\frac{\ln (n)}{2}+O(1)($ since $\sqrt{n} \geq t \geq \sqrt{n}-1)$.

Now, we deal with the second lower bound. Fix $n$ and $k$. Let $\lambda=\left\lfloor\frac{n+k}{2 k}\right\rfloor$ and $p=\lambda k$. We consider the following instance, consisting of $n$ players and $\lambda+2$ sets: $S_{0}=\{1,2, \cdots, p\}, S_{\lambda+1}=\{p+1, p+2, \cdots, n\}$ and for $i=1,2, \cdots, \lambda S_{i}=$ $\{k(i-1)+1, k(i-1)+2, \cdots, i k\} \cup\{p+1, p+2, \cdots, 2 p-k\} . S_{\lambda+1}$ has weight 0 while all other sets have weight 1 . Note that this construction is possible since $2 p-k \leq n$. Indeed, $p=\lambda k \leq \frac{n+k}{2}$.

Then the strategy profile where player $(i-1) k+j$ chooses $S_{i}$ for $i \leq \lambda$ and $j \leq k$, and player $i$ chooses $S_{\lambda+1}$ for $i \geq p+1$ is a $k$-strong equilibrium. Indeed since $S_{\lambda+1}$ has weight 0 players in $S_{\lambda+1}$ have no interest to change (as we said in Lemma 3 ), and a coalition of $r \leq k$ players (between 1 and $p$ ) changing their mind and choosing set $S_{0}$ would pay the same amount $C(p, 1, r)$ while their current cost is $C(p, 1, k) \leq C(p, 1, r)$ since the cost is non increasing with $\ell_{j}(a)$. The weight of this $k$-strong equilibrium is $\lambda$ while the Set Cover consisting of taking $S_{0}$ and $S_{p+1}$ has weight 1 .

\subsection{Uniform weights}

In Section 4.2, the lower bounds are obtained with a set of weight 0 . Then, one can wonder whether these lower bounds still hold when the weight of any set is fixed (for instance weight one). We show almost similar lower bounds for this case (Propositions 3, 4 and 5). As a final result, the PoA drops to $n / 4$ in the uniform case $(\mathrm{PoA}=0.66 n$ in the tailored model and $\mathrm{PoA}=n$ in the fair balanced model $)$.

Proposition 3. Under the tailored model, and for any $k \in[1 . . \Delta]$, the $k-S P o A$ of the SET COVER game is at least $H(k)-1+\frac{\Delta}{k}-\frac{1}{k}+\frac{1}{\Delta}$ if weights are uniform.

For $k=\Delta$ (and more generally for $k \geq \Delta$ ) this gives $H(\Delta)$. For $k=1$, the bound is $\Delta-1+\frac{1}{\Delta}$ but the next Proposition gives a better bound of $\Delta$.

Proposition 4. Under the tailored model, the PoA of the SET COVER game is at least $\Delta$ if weights are uniform.

Finally, dealing with $n$, we can also get strong lower bounds for uniform weights.

Proposition 5. Under the tailored model, and for any $k \in[1 . . \Delta]$, the $k$-SPoA of the SET COVER game is at least $\frac{n}{4 k}$ if weights are uniform.

It is also possible to get a lower bound of $\ln (n) / 2+O(1)$ for the SPoA when weights are uniform.

Proposition 5 shows that the PoA is at least $n / 4$ if weights are uniform. Here, we prove that a cost function similar to the one studied in Subsection 4.1 reaches this bound.

Proposition 6. When the local tax function is defined as $\frac{1}{s_{a_{i}}^{2}+\ell_{a_{i}}(a)}$, and weights are uniform, the PoA of the SET COVER game is at most $\frac{n}{4}+\frac{1}{2}+\frac{1}{4 n}$. 


\section{Concluding remarks}

The goal was to reduce the price of anarchy with particular local tax functions. However we did not consider converge issues. As a future work, we believe that our results should be completed with a study of the converge time to an equilibrium, depending on the tax function under consideration.

The model of local tax functions is fairly natural and captures several concrete situations where a coordination mechanism is introduced in order to reduce the social cost. Hence it should be fruitfully applied to other games (can it be applied to Anshelevich et al.'s connection game?). On a theoretical viewpoint, this work can also be seen as a way to understand to what extend relaxations of some conditions of the model (budget balance here) may improve the global efficiency. Hence the impact of other relaxations might also be investigated.

\section{References}

1. Koutsoupias, E., Papadimitriou, C.: Worst case equilibria. In: Proc. of STACS 1999, Springer LNCS 1563. (1999) 404-413

2. Aumann, R.J.: Acceptable points in games of perfect information. Pacific Journal of Mathematics 10 (1960) 381-417

3. Andelman, N., Feldman, M., Mansour, Y.: Strong price of anarchy. Games and Economic Behavior 65 (2009) 289-317

4. Rosenthal, R.W.: A class of games possessing pure-strategy nash equilibria. International Journal of Game Theory 2 (1973) 65-67

5. Holzman, R., Law-Yone, N.: Strong equilibrium in congestion games. Games and Economic Behavior 21 (1997) 85-101

6. Rozenfeld, O., Tennenholtz, M.: Strong and correlated strong equilibria in monotone congestion games. In: Proc. of WINE 2006, Springer LNCS 4286. (2006) 74-86

7. Anshelevich, E., Dasgupta, A., Kleinberg, J.M., Tardos, É., Wexler, T., Roughgarden, T.: The price of stability for network design with fair cost allocation. In: Proc. of FOCS 2004. (2004) 295-304

8. Epstein, A., Feldman, M., Mansour, Y.: Strong equilibrium in cost sharing connection games. In: ACM Conference on Electronic Commerce. (2007) 84-92

9. Christodoulou, G., Koutsoupias, E., Nanavati, A.: Coordination mechanisms. In: ICALP, Springer LNCS 3142. (2004) 345-357

10. Buchbinder, N., Lewin-Eytan, L., Naor, J., Orda, A.: Non-cooperative cost sharing games via subsidies. In: SAGT, Springer LNCS 4997. (2008) 337-349 


\section{Appendix}

Tight example for Theorem 1. Consider an instance such that $m=\Delta+1$, $w_{j}=\frac{1}{\min \{j, k\}}$ for $j=1, \ldots, \Delta, w\left(S_{\Delta+1}\right)=1, S_{j}=\{j\}$ for $j=1, \ldots, \Delta$ and $S_{\Delta+1}=\{1, \ldots, \Delta\}$. The strategy profile $a$ where $a_{i}=i$ is a $k$-strong equilibrium. Indeed, consider a coalition $N^{\prime} \subseteq\{1, \ldots, \Delta\}$ with $\left|N^{\prime}\right| \leq k$. Consider player $i=\max N^{\prime}$. If $i \geq k$, then her cost is $1 / k$ when she plays $S_{i}$, whereas this cost becomes $\frac{w\left(S_{\Delta+1}\right)}{\left|N^{\prime}\right|} \geq 1 / k$ if she chooses $S_{\Delta+1}$ (together with the other members of the coalition). If $i<k$ and her current cost is $1 / i$; it becomes $\frac{w\left(S_{\Delta+1}\right)}{\left|N^{\prime}\right|} \geq 1 / i$ since in this case $\left|N^{\prime}\right| \leq i$. In any case, it is not in that player's interest to be a member of the coalition $N^{\prime}$. Therefore we have $k S T R O N G=H(\min \{\Delta, k\})-1+\frac{\Delta}{\min \{\Delta, k\}}$ whereas $O P T=1$ if $o_{i}=\Delta+1$ for all $i$.

Lemma 3. Suppose that there exist $d \geq 1, k \geq s \geq 1$ and $w>0$ such that $C(d, 0, d)>C(s, w, s)$. Then the $k-S P o A$ of the SET COVER game is unbounded.

Proof. Consider the following instance. There is one set $S_{0}=\left\{e_{0}^{1}, e_{0}^{2}, \ldots, e_{0}^{s}\right\}$ with weight $w>0$. For every $j \in[1 . . s]$ there is a set $S_{j}=\left\{e_{0}^{j}\right\} \cup\left\{e_{j}^{1}, e_{j}^{2}, \ldots, e_{j}^{d-1}\right\}$ with weight 0 and size $d$. Some elements only appear in one set so their strategy is known for every strategy profile. The strategy profile where each element $e_{0}^{j}$ plays $S_{0}$ must be a strong equilibrium. Indeed the tax of each element $e_{0}^{j}$ which plays $S_{j}$ is $C(d, 0, d)$. If all these elements play $S_{0}$ (their number is at most $s$ ) then their cost becomes $C(s, w, s)$. Since $C(d, 0, d)>C(s, w, s)$ this deviation is profitable and possible because $s \leq k$.

The social cost of the SE is $w$ while an optimal cost is 0 , then the SPoA is unbounded.

Proposition 3. Under the tailored model, and for any $k \in[1 . . \Delta]$, the $k-S P o A$ of the SET COVER game is at least $H(k)-1+\frac{\Delta}{k}-\frac{1}{k}+\frac{1}{\Delta}$ if weights are uniform.

Proof. We consider an array of $\Delta \times(\Delta !)$ elements that we arrange in $\Delta$ lines and $\Delta$ ! columns. For $l=1, \cdots, \Delta$ !, a vertical set $S_{l}$ contains the elements of column $l$. For $l=1, \cdots, k-1$, line $l$ is partitioned into $(\Delta !) / l$ sets of size $l$. For $l=k, \cdots, \Delta-1$, line $l$ is partitioned into $(\Delta !) / k$ sets of size $k$. Line $\Delta$ is partitioned into $(\Delta !) / \Delta$ sets of size $\Delta$. These sets are called horizontal. As previously, we complete each such horizontal set by adding (at random) elements from line $\Delta$ in such a way that each horizontal set has size $\Delta$. For sets from line $l<k$ we add $\Delta-l$ elements, and for sets from line $l, k \leq l<\Delta$ we add $\Delta-k$ elements. Both vertical and horizontal sets have weight one.

Let us consider the state where every element of line $\Delta$ decides to be covered by the horizontal set of its line, while in the other lines every element decides to be covered by the (unique) horizontal set where it appears. Hence the cost incurred by an element of line $l$ is $C(\Delta, 1, l)$ for $l<k, C(\Delta, 1, k)$ for $k \leq l<\Delta$, and $C(\Delta, 1, \Delta)$ for $l=\Delta$. If $r \leq k$ elements move to the same vertical set then at least one of them does not benefit since the new cost is $C(\Delta, 1, r)$ while the lowest previous 
cost, thanks to the fact that $C$ is non increasing with $\ell_{j}(a)$, was at most $C(\Delta, 1, r)$. Then the state is a $k$-strong equilibrium.

The optimum solution (made of all vertical sets) has weight $\Delta !$. The $k$-strong equilibrium we found has weight $\left(\sum_{l=1}^{k-1} \frac{\Delta !}{l}\right)+\frac{\Delta !}{k}(\Delta-k)+\frac{\Delta !}{\Delta}=\Delta !(H(k)-1+$ $\left.\frac{\Delta}{k}-\frac{1}{k}+\frac{1}{\Delta}\right)$. Hence, the $k$-SPoA is at least $H(k)-1+\frac{\Delta}{k}-\frac{1}{k}+\frac{1}{\Delta}$.

Proposition 4. Under the tailored model, the PoA of the SET COVER game is at least $\Delta$ if weights are uniform.

Proof. Let $k$ be such that $k \geq(\Delta-1) / \varepsilon$. We build an instance with $n=k \Delta$ players and $n-\Delta+k$ sets as follows. An element is denoted by $e_{x}^{y}$ where $1 \leq x \leq \Delta$ and $1 \leq y \leq k$. There is a set $S^{y}=\left\{e_{1}^{y}, e_{2}^{y}, \cdots, e_{\Delta}^{y}\right\}$ for all $y$ in $\{1, \cdots, k\}$. There is a set $T_{x}^{y}=\left\{e_{x}^{y}\right\} \cup\left\{e_{2}^{k}, \cdots, e_{\Delta}^{k}\right\}$ for all $y$ in $\{1, \cdots, k-1\}$ and $x$ in $\{1, \cdots, \Delta\}$. All sets have weight one and size $\Delta$.

Let us show that the strategy profile $a$ where every $e_{x}^{y}$ chooses $T_{x}^{y}$ if $y \in$ $\{1, \cdots, k-1\}$ and $e_{x}^{k}$ chooses $S^{k}$ for all $x$ in $\{1, \cdots, \Delta\}$ is a NE.

The cost incurred by any element in $S^{k}$ is $C(\Delta, 1, \Delta)$. If $e_{x}^{k}$ (for $1<x \leq \Delta$ ) deviates then its cost would be $C(\Delta, 1,2)$. Since $C(\Delta, 1,2) \geq C(\Delta, 1, \Delta)$, it is not in the player's interest to deviate. Player $e_{1}^{k}$ chooses the only set where she appears. When $y<k$, the cost incurred by any element $e_{x}^{y}$ is $C(\Delta, 1,1)$. If $e_{x}^{y}$ deviates, i.e. select $S^{y}$ instead of $T_{x}^{y}$, then her cost would also be $C(\Delta, 1,1)$.

In the NE, $n-\Delta+1$ sets are selected. An optimal set cover is to select $S^{y}$ for all $y \in\{1, \cdots, k\}$. Then the ratio is $(n-\Delta+1) / k \geq \Delta-\varepsilon$.

Proposition 5. LUnder the tailored model, and for any $k \in[1 . . \Delta]$, the $k$-SPoA of the SET COVER game is at least $\frac{n}{4 k}$ if weights are uniform.

Proof. Fix some $k$ and $n$. Let $\lambda=\left\lfloor\frac{n}{2 k}\right\rfloor, p=n-\lambda k$ and $t=n-p=\lambda k$. Consider the following uniform instance consisting of $n$ players and $\lambda+2$ sets: $S_{0}=\{1,2, \cdots, p\}$ and $S_{i}=\{k(i-1)+1, k(i-1)+2, \cdots, k i\} \cup\{t+1, t+2, \cdots, t+p-k\}$ for $i=1,2, \cdots, \lambda$, and $S_{\lambda+1}=\{t+1, t+2, \cdots, n\}$. Note that $t+p-k=n-k \leq n$ and that $\lambda \leq \frac{n}{2 k}$, hence $\lambda k \leq n-\lambda k=p$, and in particular $k \leq p$. Note also that $S_{0}$ has size $p, S_{\lambda+1}$ has size $n-t=p$ and $S_{i}(1 \leq i \leq \lambda)$ has size $k+p-k=p$.

Then the strategy where player $k(i-1)+j$ chooses $S_{i}$ for $1 \leq i \leq \lambda$ and $j \leq k$, and player $i$ chooses $S_{\lambda+1}$ for $i \geq t+1$ is a $k$-strong NE. Since $C(p, 1, p) \leq C(p, 1, r)$ for $r \leq p$, players choosing $S_{\lambda+1}$ do not want to change (for $S_{0}$ ). If a coalition of $r \leq k$ players from sets $S_{1}, \cdots, S_{k}$ would change their mind and choose $S_{0}$, they would pay $C(p, 1, r)$ which is not smaller that their current cost $C(p, 1, k)$. The weight of this $k$-strong NE is $\lambda+1$ while the Set Cover consisting of taking $S_{0}$ and $S_{\lambda+1}$ has weight 2 . The ratio is then $\frac{\lambda+1}{2} \geq \frac{n}{4 k}$.

Proposition 6. When the local tax function is defined as $\frac{1}{s_{a_{i}}^{2}+\ell_{a_{i}}(a)}$, and weights are uniform, the PoA of the SET COVER game is at most $\frac{n}{4}+\frac{1}{2}+\frac{1}{4 n}$.

Proof. Let $a$ be a NE. We mainly prove that there exists a subset $N^{\prime}$ of players of size $\Delta$ (we recall that $\Delta$ is the maximum size of the sets) which choose the same set 
$S_{j}$, ie., $\forall i \in N^{\prime}, a_{i}=j$. By contradiction, for every set $S_{j}$ of size $\Delta$, let $N_{j}^{\prime}$ be the set of players which choose $S_{j}$ and let $N_{j_{0}}^{\prime}=\operatorname{argmax}\left|N_{j}^{\prime}\right|$. By hypothesis, $\left|N_{j_{0}}^{\prime}\right|<\Delta$. Hence, there is a player $k \notin N_{j_{0}}^{\prime}$ which could play $S_{j_{0}}$. We get $c_{k}(a) \geq \frac{1}{\Delta^{2}+\mid N_{j_{0}}^{\prime}}$. Actually, either $\left|S_{a_{k}}\right| \leq \Delta-1$ and then $c_{k}(a) \geq \frac{1}{(\Delta-1)^{2}+\Delta-1}>\frac{1}{\Delta^{2}}$, or $\left|S_{a_{k}}\right|=\Delta$ and $\left|N_{a_{k}}^{\prime}\right| \leq\left|N_{j_{0}}^{\prime}\right|$. Then, $c_{k}(a)>\frac{1}{\Delta^{2}+\left|N_{j_{0}}^{\prime}\right|+1}=c_{k}\left(\left.a\right|_{k} j_{0}\right)$, contradiction.

Since, $N A S H \leq 1+(n-\Delta)$ and $O P T \geq \frac{n}{\Delta}$, we deduce $P O A \leq \frac{(n-\Delta+1) \Delta}{n} \leq$ $\frac{(n+1)^{2}}{4 n}=\frac{n}{4}+\frac{1}{2}+\frac{1}{4 n}$, since the function $f(x)=\frac{(n-x+1) x}{n}$ for $x \in[1 ; n]$ reaches its maximum value for $x=\frac{n+1}{2}$. 